\title{
Suggestioni dantesche tra saudade, desío e musica pop brasiliana
}

\author{
Rosa Affatato \\ Asociación Complutense de Dantología / Universidad Complutense de Madrid \\ rosaffatato@gmail.com
}

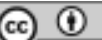

\section{Riassunto}

L'intervento prende in considerazione il concetto di saudade come analizzato nell'opera di E. Lourenço (1972) per essere poi confrontato con il desio dantesco di Pg. VIII, I-9. Sulla base dell'affermazione di A. Tabucchi a proposito della traduzione della parola e del sentimento portoghese in italiano e attraverso spunti presenti in F. Pessoa, tale confronto viene quindi applicato al contesto della nascente musica pop brasiliana degli anni '70. In particolare si fa riferimento al movimento del Tropicália, all'artista Caetano Veloso e alla band Os Mutantes, che nel 1970 ha pubblicato l'album Divina Comédia Ou Ando Meio Desligado, per arrivare fino al contesto musicale pop brasiliano odierno con l'album Dante XXI (2006) dei Sepultura e con altri esempi di irradiazioni musicali dell'opera dantesca. Il lavoro si conclude mostrando come il poema e la figura di Dante siano un esempio di "saudade attiva", sorprendentemente attuali e vicini al mondo giovanile del Brasile dagli anni '70 a oggi come e simboli di ribellione allo status quo e di libertà culturale e mentale.

Parole chiave: saudade; desío; musica pop brasiliana; Tropicália; Divina Commedia.

\begin{abstract}
The aim of this contribution is to consider the concept of saudade as analyzed in E. Lourenço's work (1972), in order to compare it with Dante's desio (Pg. VIII, I-9). This comparison, based also on A. Tabucchi's interpretation of saudade Italian translation, is applied to Brazilian pop music of the I970s. The discussion goes through F. Pessoa's poem getting to the Tropicália cultural movement, the artist Caetano Veloso and the Os Mutantes band, who in 1970 published the album Divina Comédia Ou Ando Meio Desligado. Moreover, the analysis covers some examples of today's Brazilian pop music and Sepultura's album Dante $X X I$ (2006) as cases of pop irradiations of Dante's work. The conclusion is that Dante's figure and poem are surprisingly present and near to the world of Brazilian youth since the I970s up to nowadays as symbols of "active saudade", a rebellion against the status quo and cultural and mental freedom.
\end{abstract}

Keywords: saudade; desí; pop Brazilian music; Tropicália; Divine Comedy. 
Ton c'è modo di parlare di musica e cultura di stampo portoghese senza lingua e che può significare nostalgia, malinconia, ma anche la dolcezza e la speranza che questi sentimenti portano con sé nel ricordo o nell'attesa di qualcosa che è stato o che sarà. Eduardo Lourenço, uno dei maggiori intellettuali portoghesi contemporanei, lucido e profondo interprete del contesto culturale lusitano ed europeo, in O labirinto da saudade, pubblicato nel 1972, vede la personalità del popolo portoghese come psicanaliticamente originata da una serie di "traumi" storici. Il primo viene identificato nella serie di guerre che tra i secoli IX e XI segnarono la nascita del Condado Portucalense, da quella cioè contro i musulmani per la riconquista dei territori iberici a quelle per l'autonomia del territorio di Portogallo e Galizia dalla Spagna:

O nosso surgimento como Estado foi do tipo traumático e desse traumatismo nunca na verdade nos levantámos até à plena assumpção da maturidade histórica prometida pelos céus e pelos séculos a esse rebento incrivelmente frágil [Portugal] ${ }^{2}$ (Lourenço 19925: 17).

Il secondo trauma si produsse a seguito della rovinosa sconfitta nella battaglia di Alcacer Quibir (I578), quando, piegato dai Mori e tornato a far parte del regno di Spagna per ragioni dinastiche, il Portogallo si rese conto di essere "um povo naturalmente destinado à subalternidade" (Lourenço 19925: 20), rifugiandosi in una ossessiva mitizzazione del passato e nella speranza di un mitico futuro ritorno dello sconfitto re don Sebastiano, del cui corpo non si trovò traccia sul campo di battaglia. Come espressione di quella che Lourenço chiama "ex vita" venne alla luce il poema Os Lusíadas di Luís de Camóes: "A raiz de toda a esperança, era o termos sido. E dessa ex-vida são Os Lusíadas a prova de fogo" orientandosi "para um futuro de antemão utópico pela mediação primordial, obsessiva do passado"3 (Lourenço 19925: 22). Il terzo trauma fu la perdita dell'impero coloniale nel 1975:

A quase totalidade dos principais actores da Revolução de Abril não previu, não soube medir, nem muito menos criar as condiçóes para remediar a esse espectacular traumatismo pelo simples facto de que a maioria não o pressentiu como tal, nem o percebeu na insólita e aparente apatia com que os Portugueses assistiram (se assistiram...) ao fim do seu domínio colonial (Lourenço 19925: 58).

I. I corsivi sono nell'originale.

2. "La nostra nascita come Stato fu di tipo traumatico, e da questo trauma non ci siamo mai ripresi davvero fino alla piena assunzione della maturità storica promessa dai cieli e dai secoli a questo germoglio incredibilmente fragile [il Portogallo]" (Tutte le traduzioni sono mie).

3. La radice di ogni speranza era l'essere stati. E di questa ex-vita I Lusiadi sono la prova del fuoco $[. .$.$] verso un futuro utopico di antemano per la mediazione primordiale, ossessiva$ del passato". 
Tutto ciò segna la cultura e la psicologia del popolo portoghese come nazione che riconosce la sua "autêntica situação de ser histórico em estado de intrínseca fragilidade" (Lourenço 1992 : 19)

Questa fragilità storica, questo "essere stati" e la speranza di un futuro utopico definiscono, secondo lo scrittore e filosofo portoghese, i confini della saudade come intrinseca alla cultura portoghese, dato che i portoghesi "Descontentes com o presente [...], nós começámos a sonhar simultaneamente o futuro e o passado" 4 (Lourenço 19925: 19).

Come conseguenza dei "traumi" storici di cui parla Lourenço, la saudade si è manifestata in Portogallo in diversi ambiti artistici, letterari e non, e soprattutto nella musica attraverso il fado; ma la si ritrova presente anche nelle ex colonie portoghesi con altri generi musicali affini come il samba, la bossa nova e il choro in Brasile o la morna a Capo Verde, dove grazie a Cesária Évora sappiamo che si chiama sodade.

Già nel I9I3, quando la filologa tedesco-portoghese Catarina Michaëlis de Vasconcelos affrontò il tema della saudade nel suo libro A Saudade Portuguesa, ne avvicinò il significato al vocabolo tedesco Sehnsucht, come "ricordo di aver gioito in tempi remoti, che non ritorneranno più; la pena di non poter godere della stessa gioia nel presente, o di provare piacere solo nel ricordo; e il desiderio e la speranza di poter in futuro tornare all'antica condizione di felicită" (Michaëlis de Vasconcelos 1996: 32). Di questo sentimento non sorprende che sia stato accostato a Dante anche dalla filologa tedesca, citando il commento di Francesca da Rimini alle richieste del poeta: "ricordarsi del tempo felice nella miseria" (If. V, I22-I23; cfr. Michaëlis de Vasconcelos 1996: 32). Anche secondo Lourenço "voltar-se para o passado, lembrar-se, nunca é um acto neutro", ma questa "regressão constitutiva da memória" ha varie maniere di essere vissuta nella sua relazione con il tempo, e cioè la malinconia, la nostalgia e la saudade. La malinconia "visa o passado como definitivamente passado"; la nostalgia "fixa-se num passado determinado [...] mas ainda real ou imaginariamente recuperável". La saudade "participa de uma e de outra", diventando un vero e proprio labirinto di relazione con il tempo, storicamente espresso come "excesso de amor em relaçáo a tudo o que merece ser amado: o amigo ausente, a amada distante, a natureza imemorial e íntima, escrínio de todos os amores, flor de verde pinho, ondas do mar"s (Lourenço 20I2: 92, passim). Ma con la saudade, continua Lourenço, non solo recuperiamo il passato come paradiso, ma lo inventiamo, ${ }^{6}$ in un movimento che non va più soltanto al

4. "Scontenti del presente [...], cominciammo a sognare simultaneamente il futuro e il passato".

5. Queste due espressioni fanno riferimento ad alcune delle liriche più famose della tradizione trobadorica galego-portoghese delle Cantigas de amigo: "Ai flores, ai flores do verde pino" del re trovatore Dom Dinis e "Ondas do mar de Vigo" del giullare Martim Codax.

6. "Com a saudade náo recuperamos apenas o passado como paraíso: inventamo-lo" (Lourenço 20I2: 93). 
contrario nel tempo, ma anche e soprattutto verso il futuro. Il paradiso non è solo nel passato, ma va ricostruito e inventato con una saudade attiva che non può fermarsi alla malinconia e alla nostalgia.

Nella poesia "Navegar é preciso" di Fernando Pessoa, per il quale il tempo e lo spazio non appartengono alla sfera del reale ma sono "puro esilio" (cfr. Lourenço 2006: I2O), compare una forma di questa saudade attiva. Ne riportiamo due strofe:

Navegadores antigos tinham uma frase gloriosa:

"Navegar é preciso; viver não é preciso."

Quero para mim o espirito desta frase, transformada

A forma para a casar com o que eu sou: Viver não

É necessario; o que é necessario é criar. ${ }^{7}$ (Pessoa: on line).

I "navegadores antigos" a cui si riferisce Pessoa sono i marinai di Pompeo Magno che secondo Plutarco si rifiutarono di navigare verso Roma per trasportare l'approvvigionamento di grano, e ai quali Pompeo avrebbe detto: "Navigare necesse; vivere non est necesse"8 (Plutarco 1996: 50, 2). Nel Livro do dessassosego l'autore allarga il concetto a una metafora mitologica, riprendendo il mito degli Argonauti, da intendere in senso archetipico come appartenente a un "nós [noi]" che include non solo sé stesso, ma ogni uomo: "Diziam os argonautas que navegar é preciso, mas que viver não é preciso. Argonautas, nós, da sensibilidade doentia, digamos que sentir é preciso, mas que não é preciso viver" (Pessoa 20IO: 217). ${ }^{9}$

Pessoa sostituisce in questi brani il verbo "navegar" rispettivamente con il verbo "criar" e con il verbo "sentir", creando un parallelismo tra il primo, come atto imprescindibile, preciso, della condizione storica umana, e il secondo, che la "sensibilidade doentia" mette in relazione con la saudade. "Sentir é preciso", e non è un caso che uno dei verbi che accompagna la saudade in lingua portoghese sia proprio "sentir": si dice infatti, oltre al generico "ter [avere] saudade", anche "sentir saudade". "Sentir" da una parte sostituisce il vivere, dall'altra è la causa del "navegar" come allegoria archetipica dell'azione poetica attiva e proattiva che spinge a scrivere. Si tratta di un transfert tra l'atto concreto del vergare con la penna e quello metaforico, in Pessoa ma anche in Dante, del creare poetico. Pessoa attualizza quindi la frase - "transformada" - di Pompeo coinvolgendo se stesso, uomo-poeta, come "argonauta", viaggia-

7. “Gli antichi navigatori avevano una frase gloriosa: 'Navigare è necessario, vivere non è necessario' / Voglio per me lo spirito di questa frase, dopo averne trasformata / la forma per sposarla con quel che io sono: Vivere non / è necessario, ciò che è necessario è creare".

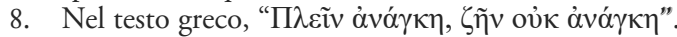

9. "Dicevano gli argonauti che navigare è necessario, ma che vivere non è necessario. Noi, argonauti dalla sensibilità dolente, diciamo che sentire è necessario, ma che non è necessario vivere" (traduzione mia). 
tore archetipico che naviga le acque della creatività: le "miglior'acque" della dantesca navicella dell'ingegno (Pg. I, I-3) che apre proprio il Purgatorio e sulla quale, lasciato il "mar sí crudele" si continua il viaggio.

Antonio Tabucchi, l'intellettuale italiano più profondamente immerso nella cultura lusitana, offrendo in una sua lettera a Remo Ceserani una propria accezione della saudade chiama in causa il Purgatorio, confrontando la traduzione italiana che di solito viene data alla parola, cioè "nostalgia" e proponendone un'interpretazione dantesca:

In italiano (come anche in altre lingue europee) la Saudade viene generalmente tradotta con 'nostalgia'. Parola inadeguata, ma soprattutto troppo giovane per un termine così antico come la Saudade. Semmai, se proprio volessimo andare in direzione nostalgica, meglio tornerebbe all'uopo il 'desío' dantesco, che nello strazio reca una tenera dolcezza, visto che quel desio (a cui l'ora volge) intenerisce il cuore a chi naviga in mari lontani. Ma della parola 'desío' si è perso l'uso. Oggi lo si chiamerebbe 'struggimento'. Forse 'magone' (Tabucchi 2003: on line).

Facendo riferimento a Pg. VIII, I-3 l'intellettuale portoghese d'adozione amplia la portata dell'aspetto emotivo-psicanalitico di questa "parola-sentimento o sentimento-parola" (D'Agostino 2006: 7) con il rivelarlo presente in nuce anche nello spazio culturale e letterario italiano. Attraverso Dante è forse possibile per i non portoghesi cercare di intendere questo aspetto della cultura lusitana come status creativo e dinamico dell'arte e della psiche, ma anche, storicamente e concretamente, per seguire l'analisi di Lourenço, della vita individuale e collettiva. Riportiamo qui le terzine iniziali di $P g$. VIII:

Era già l'ora che volge il disío ai navicanti e 'ntenerisce il core lo dì c' han detto ai dolci amici addio; e che lo novo peregrin d'amore punge, se ode squilla di lontano che paia il giorno pianger che si more; quand'io incominciai a render vano l'udire e a mirare una de l'alme surta, che l'ascoltar chiedea con mano.

Il disío dantesco è un'azione inconscia, tanto che, all'apparire delle anime della valletta di Pg. VIII, Dante incomincia a "render vano l'udire" (v. 8), cioè a non udire più nulla perché i suoi sensi coscienti, la vista e l'udito, sono presi da un'altra scena che fa immediatamente dissolvere il suono della "squilla di lontano" (v. 5) che gli era parso di sentire, non di udire realmente. Quel suono che il suo inconscio immagina e che lo "punge" (v. 5) come la "sensibilidade doentia" di Pessoa, è proprio la saudade che al "novo peregrin d'amore" (v. 4), l'argonauta-Dante, fa sentire l'urgenza di continuare il cammino intrapreso: 
deve infatti prestare attivamente attenzione a "una de l'alme surta" (vv. 8-9) che gli faceva segno con la mano, proseguendo da "visionario" il suo poema e da "visionato" il suo viaggio oltremondano. ${ }^{\mathrm{IO}}$

La saudade che muove dunque i naviganti-argonauti al navigare poetico è ciò che scrittori, artisti, studiosi chiamano a volte "ispirazione", ma altro non è che la manifestazione della spinta creativa data da questo movimento psichico che - secondo Pessoa - solo i lusitani conoscono bene, perché sono gli unici che hanno una parola per individuarlo: "Saudades só portugueses / conseguem senti-las bem / porque têm essa palavra / para dizer que as têm" (Pessoa 1997: 137). E anche se "la parola non è condizione sufficiente per la legittimazione 'antropologica' di un sentimento in quanto tale e perciò della sua esclusività" (Russo 20I3: 34I), è indubbio il fatto che, nonostante la perdita dell'impero coloniale, "il destino storico di conquiste e scoperte abbia modellato profondamente nell'anima di chi partiva e chi restava tutta una gamma di sentimenti che oggi si traducono con saudade" (Russo 2013: 339).

Il sentimento così tanto profondamente radicato nell'anima lusitana si ritrova oltremare, messo in musica dal cantautore bahiano Caetano Veloso, che insieme con Gilberto Gil fu tra gli iniziatori del movimento che portò al cambio di direzione della musica popolare brasiliana fino alla grande rivoluzione pop.

Del movimento, che dal titolo del suo album Tropicália del 1968 (Philips) prese il nome di "Tropicália" o "tropicalismo", ${ }^{\text {II }}$ e del suo orizzonte culturale che nasce come "sincretico" e antinazionalista, C. Veloso parla nel suo libro Verdade Tropical:

A palavra-chave para se entender o tropicalismo é sincretismo. Não há quem năo saiba que esta é uma palavra perigosa. E na verdade os remanescentes da Tropicália nos orgulhamos mais de ter instaurado um olhar, um ponto de vista [...] participando de uma comunidade cultural urbana individualista universalizante e internacional. Os pruridos nacionalistas nos pareciam tristes anacronismos. Ao mesmo tempo, sabíamos que queríamos participar da linguagem mundial para nos fortalecermos como povo e afirmarmos nossa originalidade. O mero aggiornamento [in italiano nel testo, n.d.A.] era pouco para nós ${ }^{12}$ (Veloso 1997: 203-204).

IO. Su Dante "visionato" e Dante "visionario" ha scritto J. Varela-Portas spiegando che Dante compie non uno, ma tre viaggi: il primo è quello di "Dante sognato o Dante visionato (nella tradizione Dante agens)"; poi quello "di ritorno cogli 'affetti sani' (Pd. XXXIII, 34-36)" e infine "il viaggio della scrittura, che compie Dante visionario [...] come personaggioscrittore" (Varela-Portas de Orduña 20I4: 339, ad loc.)

II. O movimento que, nos anos 6o, virou a tradição da música popular brasileira (e sua mais perfeita tradução - a bossa nova) pelo avesso, ganhou o apelido de "tropicalismo". O nome (inventado pelo artista plástico Hélio Oiticica [...]) Tropicália, [...] é preferível por não se confundir com o "luso-tropicalismo" de Gilberto Freyre (algo muito mais respeitável) ou com o mero estudo das doenças tropicais (Veloso 1997: I0).

I2. 2La parola chiave per intendere il tropicalismo è sincretismo. Non c'è nessuno che non 
Lo sguardo e il punto di vista universalizzanti e internazionali di cui parla Veloso portavano però anche alla contraddizione dell'apertura e della partecipazione della musica "popolare" all'industria musicale, cosa che fu fortemente contestata ai tropicalisti dall'ambiente culturale paulista, come testimoniano alcune interviste tuttora rintracciabili in Internet. ${ }^{13} \mathrm{Si}$ trattava insomma, per i tropicalisti, di riscattare la musica tradizionale dai confini eminentemente popolari e rilanciarla in maniera "sincretica", congiunta ciò̀ a influenze esterne, sia per quanto riguardava i testi, sia per gli stili musicali che senza abbandonare il samba si coloravano di influenze del rock e del pop europei:

As melodias rompiam com con os estilos establecidos e, embora trabalhadas dentro dos novos padrôes do pop internacional, traziam para perto a tradição da música nordestina, revista e aumentada. Era de certa forma tambêm uma restauração de valores musicais nacionais negados pela bossa nova e o samba-jazz, [...] reciclados e reinventados em un novo momento social e político: Luiz Gonzaga e Jimi Hendrix, os Beatles e Jackson do Pandeiro (Motta 2000: 167).

Nel 1969 Veloso, sulla scia del movimento che si andava creando nell'ambito del Tropicália, scrive, ispirandosi a F. Pessoa, un brano dal titolo non casuale: Os Argonautas, in cui il musicista riesce a mettere insieme tradizione e novità, utilizzando il genere del fado unito alle nuove tendenze melodiche della musica pop brasiliana ${ }^{\mathrm{I} 4}$ (MPB). La saudade fa da protagonista intrinseco in questo brano in cui il navigare non ha una meta né un porto che mai arriva, ma è in sé la meta del coração. Ne riportiamo le prime due strofe con il ritornello:

O barco, meu coração não agüenta

Tanta tormenta, alegria

Meu coração não contenta

O dia, o marco, meu coração

$\mathrm{O}$ porto, não

sappia che si tratta di una parola pericolosa. E in effetti quelli di noi che sono rimasti del Tropicália siamo orgogliosi di aver instaurato uno sguardo, un punto di vista [...] partecipando a una comunità culturale urbana individualista universalizzante e internazionale. I pruriti nazionalistici ci sembravano tristi anacronismi. Allo stesso tempo, sapevamo che volevamo partecipare al linguaggio mondiale per rafforzarci come popolo e affermare la nostra originalità. Il mero aggiornamento era poco per noi”.

13. Quella di un aspro dibattito organizzato il 6 giugno 1968 dagli studenti della facoltà di Architettura e Urbanismo (FAU) di São Paulo al gruppo formato da Veloso, Gil e i poeti Augusto de Campos e Décio Pignatari con il giornalista Chico de Assis si trova all'indirizzo http://tropicalia.com.br/eubioticamente-atraidos/polemica/o-tropicalismo-se-define-pelodebate (Folha:1968, on line) e riassunto ma con foto, all'indirizzo http://caetanoendetalle. blogspot.it/2013/Io/I968-debate-tropicalista.html.

I4. La "rivoluzione" in realtà era iniziata già nel 1966, quando Sérgio Mendez incise negli Stati Uniti con il gruppo "Brazil 66" la hit Mas que nada e altri brani, compresa una versione di Day Tripper dei Beatles "propondo uma jogada rítmica que integrava o samba-jazz com o pop americano e um grande repertório brasileiro" (ibidem: 157). 


\section{Navegar é preciso}

Viver não é preciso

O barco, noite no céu tão bonito

Sorriso solto, perdido

Horizonte, madrugada

$\mathrm{O}$ riso, o arco da madrugada

O porto, nada (Veloso 1969: s / p). ${ }^{\text {is }}$

I versi "O porto, não" e "O porto, nada" terminano le strofe in cui si descrive un personaggio in una barca, di notte, che "não agüenta / tanta tormenta" e che "alegria / [...] não contenta", dando voce e suono al sentimento della saudade, il cui vero protagonista è "meu coraçáo". In Pg. VIII, I-3 troviamo gli stessi elementi, cioè il disio / saudade e il core intenerito: "Era già l'ora che volge il disío / ai navicanti e 'ntenerisce il core / lo dì c' han detto ai dolci amici addio". L'elemento della "madrugada", in Veloso a prima vista positivo, è invece il contrario dell'"ora" dantesca che sarebbe piuttosto il malinconico tramonto, ma la speranza data dall'"arco da madrugada" viene subito offuscata dall'ultimo verso della strofa: "O porto, nada", finale corrispondente all'addio dantesco "ai dolci amici" che vela la terzina di saudade, del "puro esilio" di Pessoa secondo Lourenço (v. supra). Veloso riesce forse suo malgrado a mettere insieme Pessoa e Dante in un fado che contiene nel titolo il riferimento al poeta portoghese e alla mitica spedizione di Giasone in cerca del vello d'oro, come metafora della ricerca di un obiettivo anelato con saudade / sogno del futuro (v. supra, Lourenço $1992^{5}: 22$ ). Contiene però anche probabilmente un richiamo, sempre in forma di saudade, ai marinai portoghesi della spedizione di Pedro Álvares Cabral che nel XVI secolo approdarono in Brasile, co-antenati del popolo brasiliano attuale. Fu uno dei motivi che creò forti polemiche tra il Tropicália e l'ambiente culturale brasiliano di sinistra che non sopportava "nosso suposto comercialismo e, sobretudo, nosso desrespeito aos princípios do projeto estético das esquerdas, dito nacional-popular" (Veloso 1997: I43) e che vedeva la rivalutazione della cultura popolare e propria del Paese in opposizione a quella straniera e colonialista. ${ }^{16}$ Veloso e il Tropicália

I5. "La barca, il mio cuore non sopporta / tanta tempesta, allegria / il mio cuore non si accontenta. / Il giorno, il limite, il mio cuore / il porto, no. / Navigare è necessario / vivere non è necessario. / La barca, la notte nel cielo così bello / il sorriso libero, perso / l'orizzonte, l'alba / il riso, l'arco dell'alba / il porto, niente". Per ascoltarla in versione originale: https:// www.youtube.com / watch?v=IsXg-XcPowM.

I6. La polemica era iniziata con l'uscita di "Terra em transe" (I967), film di Glauber Rocha, regista, sceneggiatore e musicista bahiano leader del movimento del "Cinema Novo" e tra gli ispiratori del Tropicália, il quale aveva polemicamente indicato non solo nella trionfalismo della destra ma anche nel populismo della sinistra la responsabilità della dittatura in Brasile. Il protagonista del film, un poeta idealista di sinistra che appoggia il movimento di cambiamento politico, deluso dai candidati dei quali è sostenitore, prima un moderato, poi un progressista, durante un comizio prende la parola stigmatizzando l'ignoranza degli 
si riallacciavano invece ai principi di quello che agli inizi del secolo in Brasile era stato il cosiddetto "movimento antropofagico", iniziato da Mario e Oswald de Andrade, intellettuali di sinistra tra i fondatori del modernismo brasiliano nei primi anni '3o. Nel Manifesto Antropófago ${ }^{17}$ Oswald de Andrade esprimeva i concetti chiave del gruppo, tra i quali si allude negativamente alla saudade come ipocrisia della colonializzazione:

Encontrados e amados ferozmente, com toda a hipocrisia da saudade, pelos imigrados, pelos traficados e pelos touristes. [...] Queremos a Revolução Caraiba. Maior que a Revolução Francesa. A unificação de todas as revoltas eficazes na direção do homem. [...] Contra a Memória fonte do costume. A experiência pessoal renovada (Andrade I928: on line).

I temi del movimento antropofagico erano stati ripresi a metà degli anni ' 50 dai poeti Augusto e Haroldo de Campos e Décio Pignatari, i quali avevano lanciato il movimento della "poesia concreta", che promuoveva "uma retomada radical do espírito modernista dos anos 20 - e das idéias de vanguarda do inicio do século -, contra os pudores antimodernistas e antivanguardistas" (Veloso 1997: 147) e che stavano apprezzando i principi del tropicalismo. A questo movimento "concretista" lo stesso Veloso avrebbe fatto esplicito riferimento una decina d'anni dopo, in un brano dedicato alla città di São Paulo, Sampa (1978). ${ }^{\mathrm{I}}$

Veloso aveva scritto il brano Os Argonautas, inserito nel suo album Caetano Veloso (Philips, 1969) - scherzosamente chiamato, citando i Beatles, "White album" per la copertina completamente bianca, sulla quale appare in segno di protesta solo la firma dell'artista - in un momento particolarmente delicato della sua "navigazione" artistica, quando il movimento tropicalista era già abbastanza diffuso. Nel dicembre 1968 , in seguito infatti a un colpo di Stato interno al governo militare, il regime già dittatoriale instaurato nel 1964

operai e la loro impreparazione politica provocando l'uccisione, da parte di uno degli scagnozzi del candidato, di un "homem miserável, representante da pobreza desorganizada" che vuole ribattergli e che nel film rappresenta metaforicamente la fascia più povera della società brasiliana, quella per la quale la sinistra starebbe lottando. C. Veloso ne parla nel suo libro Verdade Tropical spiegando che "era a própria fé nas forças populares - e o próprio respeito que os melhores sentiam pelos homens do povo - o que aqui era descartado como arma política ou valor ético em si" [Era la stessa fede nelle forze popolari e lo stesso rispetto che i migliori sentivano per la gente del popolo che qui era scartato come arma politica o valore etico in sé]. (Veloso 1997: 68).Il film è disponibile su Youtube: https://www.youtube. $\mathrm{com} /$ watch? $\mathrm{v}=\mathrm{bX} 4 \mathrm{BLrGwWGQ}$ (rev. I2/Io/20I6).

17. Il nome del movimento alludeva ironicamente a un episodio della storia brasiliana, riportato in calce al Manifesto: il naufragio della nave sulla quale viaggiava un vescovo portoghese, seguito dalla morte del vescovo stesso, divorato da indios antropofagi.

I8. Il brano è incluso nell'album Muito (Universal Music Japan, 1978). Riportiamo il verso in cui descrivendo la città con la quale aveva avuto un approccio difficile dice: "É que quando eu cheguei por aqui eu nada entendi / Da dura poesia concreta de tuas esquinas/ da deselegância discreta de tuas meninas ( $\grave{E}$ che quando arrivai qui non capii nulla / della dura poesia concreta dei tuoi angoli / della diseleganza discreta delle tue ragazze)". 
da Castelo Branco aveva virato verso un inasprimento e Veloso, insieme con l'amico Gilberto Gil, erano stati arrestati subito dopo perché considerati pericolosi a causa della portata rivoluzionaria del movimento musicale di cui erano esponenti. Veloso racconta che dopo la cattura si trovava con l'amico agli arresti domiciliari a Salvador prima di essere esiliati entrambi a Londra e in quel periodo entrambi incisero, con gli scarsi mezzi a disposizione, ciascuno un nuovo disco ${ }^{19}$ e quello di Veloso fu proprio il "White album". Il brano Os Argonautas nasce dunque in tale frangente per raccontare la saudade di una libertà perduta senza prospettiva di essere presto recuperata, ma ciononostante senza lo scoraggiamento eventualmente provocato dalla situazione in cui il musicista si trovava e anzi con la convinzione che la lotta e la protesta dei "naviganti", veicolate dal naviglio della musica, avrebbero sortito l'effetto di miglioramento sociale auspicato dai poeti "concreti" e dal movimento antropofagico.

Nello stesso periodo anche altri musicisti pop brasiliani legati al tropicalismo stavano trasformando in protesta l'orizzonte della saudade con quella che Tabucchi, sulla scia di Lourenço, chiama "nostalgia del futuro":

[...] il padre gesuita António Vieira, fecondissimo prosatore e predicatore, strenuo paladino degli Indios brasiliani, uomo di Chiesa e di mondo [...], elaborò un trattatello storico (o di fantastoria), História do Futuro [...]. Con Vieira i portoghesi smisero di avere Saudade di una grandezza passata che si ostinava a non tornare, e si misero a praticare la nostalgia del futuro (Tabucchi 2003: on line).

Il futuro del Brasile era orientato alla caduta della dittatura - che cadde molto più tardi, nel 1985 - e come "bandiera" della protesta musicale venne scelto, da Os Mutantes, una delle band più in voga del momento, proprio Dante.

Anche i Mutantes fin dall'inizio della loro attività musicale manifestarono una "postura contracultural [...]. Misturando ritmos, sonoridades; renovando [...] também através de instrumentos diferentes e letras que brincam com as palavras e as possibilidades musicais" inserendosi nello scenario di "mudança, rompimento e psicodelia tropical" (Witzel - Costa 20I3: 2) fino a diventare un vero e proprio simbolo del rock brasiliano degli anni '70.

Il loro album Divina Comédia ou Ando meio desligado esce nel 1970 per l'etichetta tedesca Polydor ed è una ricerca di nuovi timbri e sonorità che suscitò

19. Lo racconta Veloso: "Gil e eu fizemos, cada um de nós, um disco nesse meio tempo. Como não podíamos ir ao Rio ou a São Paulo, fizemos as gravaçôes num estúdio pequeno em Salvador (acho que se chamava Estúdio J.S.), apenas com violáo (Gil e io facemmo, ognuno di noi, un disco in questo frattempo. Siccome non potevamo andare a Rio o a Sao Paulo, facemmo le registrazioni in un piccolo studio a Salvador (penso si chiamasse Studio J.S.), con la sola chitarra)" (Veloso 1997: 417). Le registrazioni di chitarra e voce furono successivamente inviate all'amico tropicalista Rogério Duprat perché vi aggiungesse gli altri strumenti e arrangiamenti. 
considerevoli polemiche e scandalo per i "modernismi" che si scontravano con l'élite conservatrice: ritmi presi dal blues, dal rock e dal dixieland per arrivare a un rock progressivo attraverso "intervençóes eletrônicas e novas timbragens instrumentais [...] mais desenvolvidas e evidenciadas" (Costa 2006: 58). Lo scandalo cominciava già dalla copertina dell'album, che originariamente prevedeva una foto a colori dei tre componenti del gruppo ritratti insieme in un letto. Tale foto fu però abbandonata e sostituita da una in bianco e nero che riproduce un personaggio - che a prima vista sembra femminile - che si erge "dalla cintola in su" (If. X, 33) fuori da un sarcofago, mentre altri due personaggi ammantati di nero la osservano seri. Il riferimento dantesco al canto di Farinata degli Uberti è evidentissimo: i tre membri del gruppo, che aveva collaborato con Veloso nell'album-manifesto Tropicália, vollero ritrarsi come personaggi della Divina Commedia, riproducendo fotograficamente nella loro copertina l'incisione di Gustave Doré dedicata a Farinata. Le due immagini combaciano quasi perfettamente. Sulla copertina dell'album il personaggio che corrisponde a Farinata viene rappresentato nella stessa posizione dell'incisione di Doré, con una figura che emerge dal sepolcro e si protende verso l'alto. La carica emotiva della rappresentazione, a giudicare dal contenuto del disco, non può essere che di protesta, in ideale parallelismo con il "gran dispitto" che Farinata prova nei confronti dell'inferno: "ed el s'ergea col petto e con la fronte / com'avesse l'inferno a gran dispitto" (If. X, 35-36). Per rappresentare il personaggio fu scelta però una raffigurazione che sembra femminile $\mathrm{o}$ androgina, forse la stessa Rita Lee, unica componente femminile del gruppo formato, oltre a lei, da Arnaldo e Sérgio Dias Baptista, quest'ultimo il chitarrista del gruppo. ${ }^{20}$ Per gli altri due personaggi, in Doré Dante e Virgilio, si possono notare parallelismi e rovesciamenti rispetto alla foto dell'album. Il Dante di Doré [Fig. I] sembra spaventato dall'emergere di Farinata e si volta dall'altra parte, mentre sulla copertina del disco [Fig. 2] i due personaggi sembrano non aspettare altro che la donna / androgino faccia la sua uscita dal sepolcro. Si può parlare di un contrappasso "moderno" in cui la corrispondenza non è tra colpa e pena, ma tra status e movimento, cioè tra la società oppressa dalla dittatura e l'anelito alla liberazione. L'immagine dell'album propone infatti, al contrario del Dante di Doré, che si ritrae spaventato, due personaggi che non provano affatto spavento per la circostanza. I personaggi che dovrebbero essere Dante e Virgilio sulla copertina del disco non presentano attributi distintivi, anzi non sono identificabili come l'uno o l'altro, lasciando così al viaggiatore e alla guida un unico ruolo scambievole nel viaggio verso la libertà. Potremmo

20. C'era anche un terzo fratello, Cláudio, proprietario di una propria marca musicale, la CCDB (Cláudio César Dias Baptista) che mantenne in attività fino a metà degli anni ' 90 e che nei Mutantes si occupava della parte sonora e meccanica delle chitarre che costruiva egli stesso (cfr. Paiva 2006: 497-498). 
dire cioè che questi due personaggi, non più spaventati, sono "nós, argonautas" di cui parlava Pessoa e che nell'immagine sembrano non aspettare altro che l'androgino, uomo e donna, dunque rappresentante della società intera, esca fuori dai propri nascondigli per unirsi a loro e unirsi alla protesta dei giovani diciannovenni Mutantes che si esprime con l'unico mezzo possibile, cioè la musica, trovando in Dante una forma di supporto culturale.

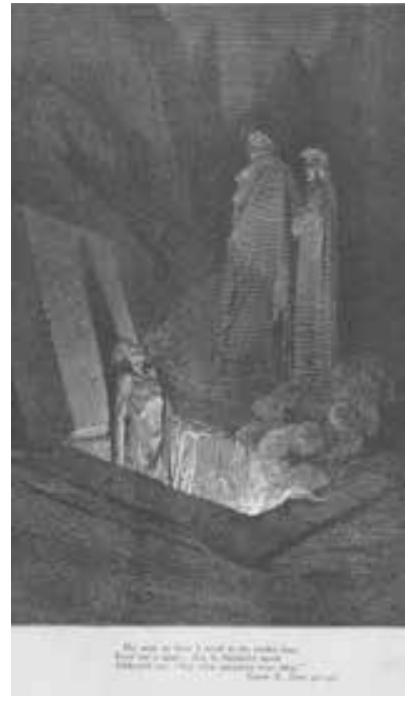

Figura I

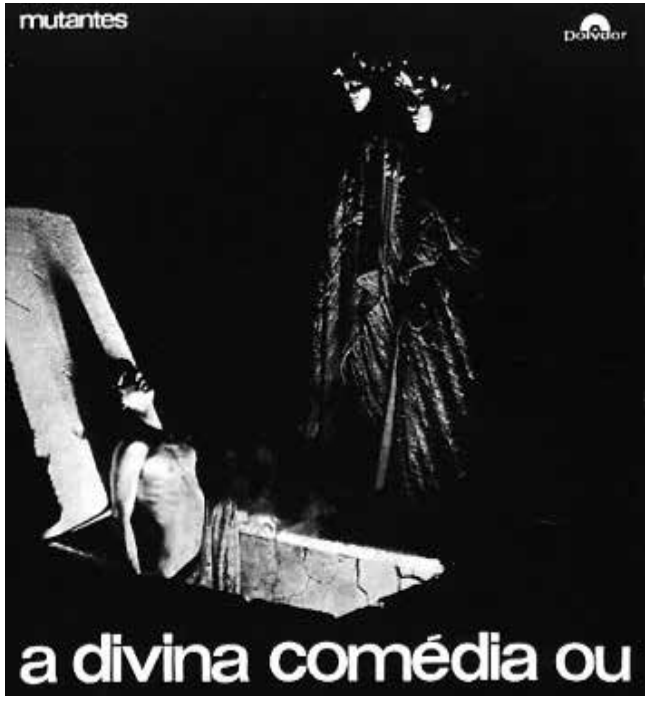

Figura 2

Emerge da questa analisi la presenza di Dante nella cultura brasiliana negli anni '70 e la sua importanza come baluardo della libertà. Dante è esule per motivi politici, proprio come molti giovani brasiliani (Veloso e Gil sono solo un esempio) in quegli anni, ed è poeta, quindi artista come loro. Un Dante molto vicino ai giovani, capace di farsi portatore delle istanze di protesta sociale del Brasile e figura con cui i giovani esponenti del movimento tropicalista potevano identificarsi. Il sottotitolo del disco Ando meio desligado può tradursi con "Mi sento un po' dissociato" e fa riferimento al rock progressivo che rompeva con la tradizione musicale brasiliana dell'epoca sia per lo stile, sia per l'uso di strumenti elettrificati, facendo sì che il disco in Brasile "had no point of reference until almost 30 years later" (Wiznitzer: on line). ${ }^{2 \mathrm{I}} \mathrm{Ma}$ il riferimen-

2I. I Mutantes riuscirono a trasformare la scarsezza di mezzi tecnici a disposizione in creatività. Nel Brasile degli anni '7o gli strumenti elettrificati e i loro accessori erano pochi e rudimentali, certo non all'altezza di quelli usati nel rock nordamericano e inglese al quale si ispirava il movimento tropicalista. I Mutantes, grazie all'inventiva dei fratelli Baptista, superarono questo scoglio creando dei propri e personali effetti sonori in sostituzione per esempio dei pedali o modificando la chitarra Regvlvs a cui Cláudio aggiunse ben sette effetti di distorsione separati, imitando le Roland che in Brasile erano ancora quasi introvabili (cfr. 
to è anche, e non secondariamente, alla "dissociazione" civile nei confronti del governo e della società che lo appoggiava. Tali sentimenti espressi dal titolo e dall'immagine esprimono a nostro parere la saudade del futuro, il desiderio di trasformazione che attraverso una rivoluzione musicale dai nuovi contenuti e nuovi strumenti manifesta le aspirazioni al cambiamento che i Mutantes portano nel nome che avevano scelto di darsi. Ma fa riferimento anche al significato semantico sia del loro nome proprio, Mutantes, participio presente del verbo mutar, di cui tranformar, verbo usato da Pessoa nella poesia che si è citata, è sinonimo, sia a quello dell'espressione "divina comédia" che in portoghese equivale a "gran confusione", in riferimento all'Inferno e parallelamente al momento politico che il Brasile stava vivendo. Insomma, questa giovane band stava mettendo in pratica, con il verbo-nome al participio presente, la trasformazione dal navegar al criar, operando la transizione dalla saudade del passato a quella del futuro; e il risultato creato attraverso la musica era qualcosa di assolutamente nuovo.

Il brano che dà il titolo all'album è quello più conosciuto e mostra come la scia del rock nordeuropeo e statunitense non si limitasse all'uso della chitarra elettrica che qui propone assoli con varie distorsioni, ma comprendesse anche l'organo Hammond e la voce di Rita Lee che riproduce i glissati vocali della grande interprete statunitense Janis Joplin. La prima strofa del brano esprime la dissociazione del protagonista rispetto al mondo a causa di un amore non ancora dichiarato:

\section{Ando meio desligado}

Eu nem sinto meus pés no chão

Olho e não vejo nada

Eu só penso se você me quer (Os Mutantes I970: s/p). ${ }^{22}$

Il brano prosegue e termina richiamando il senso di confusione come allontanamento dalla realtà in direzione di un'introversione mentale da parte dell'amante che pensa continuamente all'amata e ha imparato a memoria ciò che ha da dirle ("Eu nem vejo a hora de lhe dizer / Aquilo tudo que eu decorei") nella speranza di ottenere il bacio immaginato ("E depois o beijo que eu já sonhei") ma nel timore che l'amata se la prenda a male ("Por favor, não leve a mal"):

Eu nem vejo a hora de lhe dizer

Aquilo tudo que eu decorei

E depois o beijo que eu já sonhei

Você vai sentir, mas...

Por favor, não leve a mal

Paiva 2006: 499).

22. "Mi sento un po' dissociato / non sento neanche i piedi per terra / guardo e non vedo nulla / penso solo se tu mi ami". 
Eu só quero que você me queira

Não leve a mal (ivi). ${ }^{23}$

L'album Divina Comédia si apre dunque con un tema stilnovistico quasi cavalcantiano, quello dell'amore-timore che allontana dalla realtà, proseguendo poi nello stesso brano con l'intenzione di iniziare un percorso, un viaggio, verso la ricerca della donna amata, in una sorta di rovesciamento rispetto alla Commedia dantesca in cui è invece Beatrice a prendere l'iniziativa per il viaggio di Dante. ${ }^{24}$ Un brano molto più complesso, ispirato non solo alla mela tentatrice come simbolo di liberazione sessuale ma anche al simbolo di Lucifero come angelo caduto e ribelle, è Ave, Lúcifer, in cui alcune sonorità sono ispirate ai Beatles di Sergent Pepper's in un testo abbastanza articolato che vede un Lucifero tra inferno e paradiso terrestre, un vero "Éden infernal", come viene chiamato nel brano, in un quadro che è metafora del sistema politico:

As maçãs envolvem os corpos nus

Nesse rio que corre

Em veias mansas

Dentro de mim

Anjos e arcanjos

Não pousam neste Éden infernal

$\mathrm{E}$ a flecha do selvagem

Matou mil aves no ar

Quieta, a serpente

Se enrola nos seus pés

É Lúcifer da floresta

Que tenta me abraçar

Vem amor

Que um paraíso

Num abraço amigo

Sorrirá pra nós

Sem ninguém nos ver

Prometo

Meu amor macio

Como uma flor

Cheia de mel

Pra te embriagar

Sem ningém nos ver

23. "Non vedo l'ora di dirle / tutto quello che ho imparato a memoria / e poi il bacio che ho sognato / tu lo sentirai... ma / Per favore, non prendertela a male / voglio solo che tu mi $\mathrm{ami}$ / non prendertela a male".

24. Per ascoltare la versione originale: https:// www.youtube.com/watch?v=1QyH4QSLNdg (rev. 7/Io/20I6). 


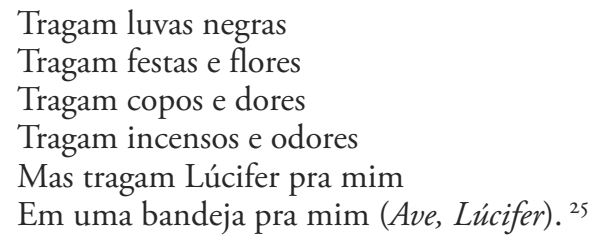

La metafora, che si avvicina a un'allegoria, oltre ad alludere alle proteste sessantottine e ai movimenti per l'autodeterminazione personale e sessuale va letta infatti come protesta contro l'inferno della dittatura. La scelta del personaggio di Lucifero si inserisce nel contesto di opposizione culturale e politica come figura ribelle ai divini disegni immutabili (cfr. Goffman - Joy 2007: 28) e quindi anch'egli simbolo dell'opposizione allo status quo. L'immagine che il testo propone di un Lucifero da portare "su un vassoio" (d'argento, evidentemente) tra incensi e profumi è una chiara allusione biblica alla testa del Battista, richiesta da Salomè, ma i ruoli dei personaggi appaiono rovesciati, perché Salomè, nella voce femminile della Lee, nel testo biblico chiede a Erode di mettere a tacere il profeta e decapitarlo, mentre qui il Lucifero ribelle rappresenta l'unica salvezza per la società afflitta dal dittatore / falso profeta da mettere a tacere attraverso i vari movimenti di opposizione, di cui il Tropicália era uno dei principali esempi. ${ }^{26}$

La saudade fa capolino nel brano sia nel ritmo rock andante, sia nel tema del rimpianto ma anche dell'aspirazione a una futura liberazione sociale e politica.

Il percorso dantesco prosegue con brani che richiamano il viaggio verso il paradiso come "Chão de Estrelas" (pavimento di stelle) che prende come spunto un popolare omonimo brano della musica tradizionale seresta di Oreste Barbosa e Silvio Caldas, ma rivisitandolo con strumenti e suoni che parodiano l'originale trasformandolo in un "pastelão sonoro" (cfr. Calado 1996: 218): rumori di aereo, spari, versi di animali e orologio a cucù, chicchirichì. Il brano

25. "Le mele avvolgono i corpi nudi / in questo fiume che scorre / in vene quiete / dentro di me. / Angeli e arcangeli / non si fermano in questo Eden infernale / e la freccia del selvaggio / ha ucciso mille uccelli nell'aria. / Un serpente, quieto, si arrotola ai suoi piedi / e Lucifero, dalla selva / tenta di abbracciarmi. / Vieni amore / che un paradiso / in un abbraccio amico / sorriderà per noi / Senza che nessuno ci veda / Prometto / il mio amore soave / come un fiore / pieno di miele / per ubriacarti / senza che nessuno ci veda. / Portate guanti neri / portate festa e fiori / portate coppe e dolori / portate incensi e profumi / ma portatemi Lucifero / su un vassoio per me". Per ascoltarla in versione originale: https://www. youtube.com/watch?v=D4ZaMNj4u5w, rev. 7/10/2016).

26. Ricordiamo che pochi anni prima, nel giugno 1968, gli esponenti del tropicalismo avevano partecipato alla "Passeata dos Cem Mil", una manifestazione di protesta di sei ore contro la dittatura che coinvolse circa centomila studenti ed esponenti del mondo culturale, sacerdoti e artisti carioca per il centro di Rio de Janeiro, repressa dalla polizia (Veloso 1997: 240, UOL 1968: on line). L'evento fu seguito pochi mesi dopo dal colpo di stato e nell'inasprimento del regime nel dicembre 1968. 
fu, secondo C. Calado, il principale responsabile dello scandalo e delle polemiche suscitate dal disco, che fu addirittura simbolicamente spaccato a metà durante uno spettacolo televisivo condotto dal critico musicale Flávio Cavalcanti, "figura folclórica do conservadorismo sensacionalista" (Veloso 1997: I43) e già uso a tali azioni spettacolari, ${ }^{27}$ dopo un infiammato discorso contro la decadenza dei valori della gioventù. Un pavimento di stelle che fa anch'esso discutere, dunque; nulla era paradisiaco nella società brasiliana, secondo i Mutantes, e le reazioni a questo brano lo hanno dimostrato.

Il penultimo brano, "Haleluia", tutto strumentale, inizia con un organo e un coro di voci maschili che cantano quest'unica parola, alludendo inizialmente alla musica religiosa ma aggiungendo in seguito piano, chitarra elettrica e basso e ottenendo ancora una volta un ibrido, "um cruzamento da música litúrgica com a profana" (Costa 2006: 58). Si può riconoscere qui la saudade dell'attesa, il desio che congiunge la tomba di Farinata con la caverna dei "beati al novissimo bando" che nel paradiso terrestre di Pg. XXX, I3-I5 "surgeran presti ognun di sua caverna / la revestita voce alleluiando". I beati / Mutantes, contaminando inferno ed Eden, mostrano l'altra faccia della medaglia, proponendo una società in cui forse qualcosa può ancora cambiare con l'unione degli sforzi che, come succede nel brano, devono mescolarsi e umanizzarsi senza relegare il cambiamento a un'opzione della bontà divina. Bisogna "tornare al mondo" (cfr. Pg. V, I30) e prendere sul serio i propri impegni politici e sociali. ${ }^{28}$

L'ultimo brano del disco svela la delusione del progetto iniziale del viaggio di ricerca dell'amata. "Oh, Mulher Infiel" è aperto da un assolo di batteria e grida, seguito da un assolo di chitarra elettrica con distorsioni e dissonanze, mentre il testo parla del "jeito" (modo di agire) e del "peito saliente" (petto prosperoso) della donna "traiçoeiramente ativa" che ha consumato la vita dell'amante:

\author{
Oh! mulher infiel \\ Traiçoeiramente ativa \\ Com minha vida consumida \\ Pelo teu jeito \\ Pelo teu peito saliente \\ Eficiente nas horas vivas \\ E nas horas vagas-pagas \\ Oh! mulher infiel (Os Mutantes I970: s/p) 29
}

27. Veloso racconta che anche il suo disco "Alegria alegria" fu spaccato a metà nel 1968 dallo stesso Cavalcanti perché a suo parere conteneva allusioni all'acido lisergico nelle lettere iniziali delle parole "(sem) Lenço, Sem Documento" nel testo della canzone (Veloso 1997:I43).

28. Versione originale: https://www.youtube.com/watch? $=$ fhqMp8rgJdg (rev. 7/10/2016).

29. "Oh! Donna infedele / tradimentalmente attiva / con la mia vita consumata / dal tuo modo di fare / dal tuo petto prosperoso / efficiente nelle ore vive / e nelle ore pigre-pagate / Oh! Donna infedele". Per ascoltarla in versione originale: https://www.youtube.com/ watch?v=Waais_kvBfY (rev. 7/10/2016). 
Il testo rende l'immagine della delusione amorosa come metafora del tradimento degli ideali rispetto a una società / falsa madre che nell'immagine del petto traditore non comprende la voglia e la necessità del cambiamento, ancorata com'è alla saudade passiva della nostalgia.

Non è da sottovalutare, infine, in tutto l'album, il ruolo della Divina Commedia come simbolo di opera erudita e popolare al tempo stesso, capace di rappresentare il superamento dei limiti tra la musica colta e quella pop, indicato dai tropicalisti con un neologismo di Pignatari, il "prodossumo", 30 ottenuto congiungendo i termini "produção" e "consumo" per evidenziare la non contraddizione degli stili poetici e musicali ai quali si ispirava tutto il Tropicália.

L'ispirazione a Dante come poeta nella musica pop brasiliana non si esaurisce con gli anni '7o. Un esempio significativo che prende spunto dalla Divina Commedia come esperienza amorosa si deve infatti al cantautore Belchior, che nel suo brano Divina comédia humana, tratto dall'omonimo album del I99I (ed. Movie Play), "umanizza" il viaggio dantesco come itinerario individuale di una vicenda sentimentale che porta confusione e angoscia ma che è pur necessario attraversare:

Quando você entrou em mim como um Sol no quintal

Aí um analista amigo meu disse que desse jeito

Não vou ser feliz direito

Porque o amor é uma coisa mais profunda que um encontro casual $[\ldots]$

Eu quero gozar no seu céu, pode ser no seu inferno

Viver a divina comédia humana onde nada é eterno

Ora direis, ouvir estrelas, certo perdeste o senso

Eu vos direi no entanto:

Enquanto houver espaço, corpo e tempo e algum modo de dizer não

Eu canto (Belchior I99I: $s / p){ }^{31}$

Il senso di precarietà dato dall'aggettivo "umana" riferito alla divina comédia rende l'opera dantesca per Belchior il paradigma della vita, cioè un viaggio tra il "cielo" e l'"inferno" dell'amore, per rispondere alla cui precarietà l'autore non trova altro strumento che il canto, suono della poesia ${ }^{32}$. "Eu canto", conclude

30. Il neologismo di Décio Pignatari si riferisce alla "ruptura dos limites entre música erudita e popular" (Campos 1978: 27I), e di conseguenza anche alla musica come oggetto di diffusione e di consumo.

3I. "Quando sei entrata in me come un sole nel cortile / Un analista mio amico mi disse che in quel modo / Non sarei stato mai felice / Perché l'amore è una cosa più profonda di un incontro casuale / [...] Ciò che voglio è godere nel suo cielo, o magari nel suo inferno / Vivere la divina commedia umana dove nulla è eterno / Mi direte, ascoltare le stelle, davvero hai perso la ragione / Io vi dirò nel frattempo: finché c'è spazio, corpo e tempo e qualsiasi modo di dire no, io canto". Per ascoltare la versione originale: https://www.youtube.com/ watch?v=nPbTs9ZJcfU (rev. 7/ro/2016).

32. All'epoca della canzone, il cantautore aveva iniziato una sua traduzione della Divina Commedia in portoghese (Bortolotti 2013: on line). Il cantante è attualmente sparito dalla cir- 
infatti il brano: l'obiettivo sembra essere proprio il "cantar", il cui percorso attraverso "espaço, corpo e tempo" è raffinato in tutto il brano dalla saudade.

Ancora in questi ultimi anni una giovane band di Rio de Janeiro nata nel 2004, gli Scracho, ha inserito nel suo primo album $A$ grande bola azul (etichetta indipendente, 2007) il brano Divina Comédia in cui, attraverso il tema amoroso, si fa riferimento a quelli che sono diventati i luoghi comuni del poema: "Com você eu faço do inferno o paraíso" (Con te io faccio dell'inferno un paradiso), si canta nel ritornello, e questo a causa del sorriso della donna amata che, in un mondo in cui il purgatorio è un "intervallo tra il cielo e la terra", porta il giovane fino al "paradiso". Riportiamo la terza strofa del brano, in cui si allude a Dante a piene mani, compresa la canzone Amor che movi (Rime, XC), probabilmente rifacendosi da una parte al tema dantesco dell'amore per la "pargoletta" 33 vista come oggetto d'amore adolescenziale, dall'altra identificando, probabilmente senza averne cognizione, la "pargoletta" con "Bia", Beatrice:

E assim como Dante por Bia por causa do seu sorriso

Eu sigo pro inferno e volto ao paraíso

Só pra poder te ver, só pra te encontrar

A tua estrela é o que me guiará

"O amor me move" só por ele eu falo

Sem ter você o purgatório é o intervalo

Entre o céu e a terra, entre o ter e não te ter

Não há distância que me faça te esquecer (Scracho 2007: s / p). ${ }^{34}$

Questi versi, in ritmo reggae, riassumono il punto di vista di un gruppo di adolescenti, da poco diplomatisi in una scuola superiore di Rio, i quali per parlare di amore e innamoramento scelgono Dante come punto di riferimento. Dante è cioè riuscito a inserirsi nella variegata multiculturalità delle grandi città del Brasile e nella formazione intellettuale dei suoi giovani come segno della cultura italiana emergendo attraverso una fusione tra la musica pop e la poesia. I testi di un autore letterario non contemporaneo e non brasiliano

colazione dal 2009, perché ricercato dalla polizia per questioni finanziarie causate da una relazione amorosa che lo ha spinto a lasciare la moglie con cui era sposato da 35 anni (ivi).

33. "[...] il poeta ama una 'pargoletta' tanto giovane e sentimentalmente immatura che non solo non è in grado di corrispondere al suo amore ma addirittura non riesce a percepire di che si tratti, sì che a lui non resta altro che aspettare, augurandosi che in futuro, avanzando nell'età, anche lei finisca per subirne la forza. [...] E [ci si chiede] se sia la "giovane» che proprio per "giovanezza» conduce a morte il poeta senza neppure accorgersi "com'ella piace I né com'io l'amo forte» della canzone Amor che movi (vv.25 e 57-9)" (Fenzi 20II: 20).

34. "E così come Dante con Bea, a causa del suo sorriso / io continuo attraverso l'inferno e torno verso il paradiso / solo per poter vederti, solo per incontrarti / la tua stella è quella che mi guiderà / "L'amor mi move", solo per lui io parlo / senza avere te il purgatorio è l'intervallo / tra il cielo e la terra, tra l'averti e il non averti / non c'è distanza che mi faccia dimenticarti”. Per ascoltare la versione originale: https://www.youtube.com/ watch?v=nIFil2oKTSw (rev. 7/10/20I6). 
sono riusciti a fondere piacevolmente e con leggerezza il desio (d'amore) con la saudade in un incontro che riflette l'identificazione dei due sentimenti espressa da Tabucchi all'inizio di questo intervento. Dante è entrato nel processo di identificazione degli adolescenti brasiliani attraverso la musica mescolandosi con il loro mondo, oggi come negli anni ' 70 , incarnando tematiche come la libertà e l'amore, fondamentali nel processo di crescita psicologica e sociale proprio della fase adolescenziale. 35

Ma la schiera dei seguaci di Dante nella musica brasiliana attuale non finisce qui. L'ultimo esempio è quello dei Sepultura, notissimo gruppo heavy metal che nel 2006 ha lanciato l'album Dante XXI (Steamhammer / SPV) il cui contenuto è, come promette il titolo, basato sulla Divina Commedia nel XXI secolo.

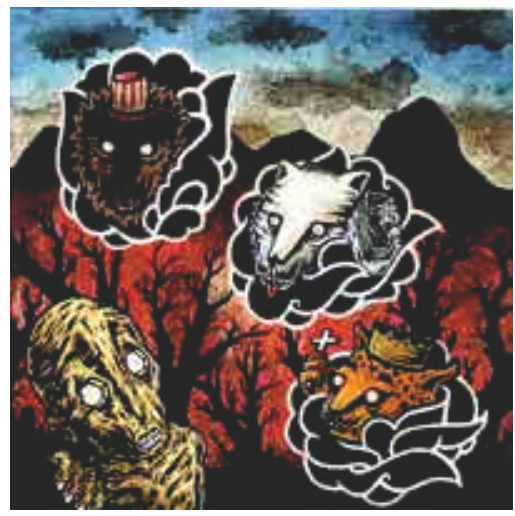

Figura 3

Anche se, tra gli appassionati del genere, vi è chi ha giudicato il disco "poco incisivo" (Nunziata 2006: on line), ciò che interessa è che la carica di protesta incarnata dal genere metal abbia visto in Dante un esempio da poter riproporre nel nostro secolo come personaggio impegnato nel denunciare i mali della società. Il disco segue la scansione dell'opera dantesca e dedica i brani a inferno, purgatorio e paradiso in ordine numerico, proprio come i canti nel poema, introducendo le varie parti (corrispondenti alle cantiche) con pezzi strumentali. Si immagina un protagonista, naturalmente Dante (ritratto sulla copertina del disco) [Fig. 3], che percorre i diversi brani e che parla in prima persona "riscrivendo" il poema e dunque viaggiandovi attraverso. L'Inferno, prima parte dell'album, si apre con Lost, brano strumentale riferito a Dante perso nella selva oscura, preludio a The Dark Wood of Error, in cui si fa riferimento alle tre fiere: "Three beasts blocked the path / That lead the way to my life" (Sepultura 2006: s / p), due delle quali sono interpretate come il

35. Ci proponiamo un approfondimento su questa tematica in un prossimo lavoro. 
Regno Unito e gli Stati Uniti ("The beast from the UK / The beast from the US”, ivi). L'allusione è al capitalismo e al predominio ideologico e politico mondiale dei due Paesi citati e rappresentati in una delle immagini del booklet del disco: la lonza con una corona sulla testa (UK), il leone con il cilindro di Uncle Sam (USA) e la lupa con un mazzetto di dollari [Fig. 4]. Le tre fiere, una delle allegorie dantesche più conosciute, sono rivisitate e intese in chiave attuale, proponendo un contenuto di dissenso che appare come risultato di un'analisi non certo superficiale del poema dantesco.

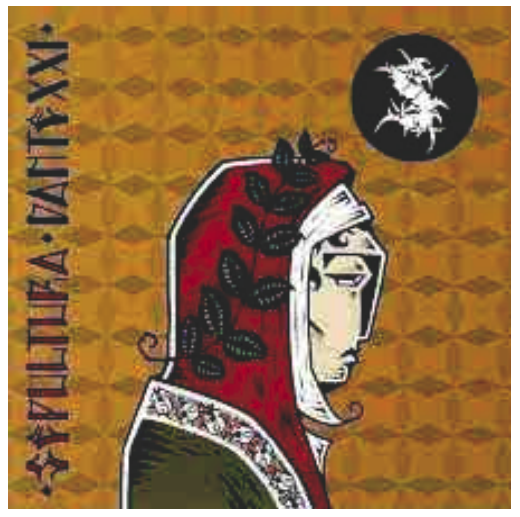

Figura 4

Anche per il Purgatorio è possibile individuare la stessa operazione "ermeneutica". Il brano introduttivo strumentale è Limbo, a cui segue Ostia, in cui si svela inizialmente la scena di Pg. I: "The skies are open before me / The crowd of souls in sudden fight / Hoping for prayers in the world" (ibidem), che allude all'affollarsi delle anime intorno a Dante per ottenere preghiere quando tornerà nel mondo. Ma questa scena quasi idillica di "cieli aperti" è immediatamente rotta dal ricordo di ciò che Dante ha appena lasciato, cioè i "traditori del popolo" nella bocca di Lucifero che per questo motivo "sono senza faccia", o come si potrebbe dire in italiano, "sfacciati": "I thought the worst had past / Traitors of the people, they have no face" (ivi) sui quali Dante fa una scoperta sconvolgente: "Those fools are the ones we vote for / The kings and rules of negligence" (ivi), per cui afferma che "I have to be cleansed, from all the blame" (ivi): dovrà cioè percorrere l'itinerario di purificazione nel purgatorio perché scopre di aver finora collaborato con le scelte politiche di questi "fools"e "traitors of the people", perché essi sono "the ones we vote for".

Non commentiamo per ragioni di spazio tutti i brani dell'album, ma precisiamo che alla fine si apre uno spiraglio di speranza: "There is a way out", si recita in "Crown and Miter", dove, dopo il terzo brano strumentale "Eunoè", dedicato al paradiso terrestre, Dante afferma ciò che ha imparato al termine 
del suo viaggio, riuscendo a vedere il bene compiuto grazie all'immersione nel fiume purgatoriale: "I had to learn, salvation / To find my place in the world we live / I had to have, compassion / To understand that I have to give / There is a way out / Salvation!!” (ibidem), in cui salvezza e compassione sono gli strumenti per trovare il proprio posto nel mondo ma al di fuori dai sistemi sociali e politici che stringono l'uomo in scelte troppo spesso imposte da politiche nazionali e sovranazionali. Per il Paradiso si offrono infine solo brani strumentali, Primum mobile e Still Flame, quasi a dire che giunti a quel punto "non eran da ciò le proprie penne" ( $P d$. XXXIII, I39) e l'unica possibilità di linguaggio è la musica. Anche attraverso il genere musicale progressive il gruppo brasiliano esprime una fortissima voglia di reazione alla società del nostro tempo. Siamo di fronte a un'attualizzazione del poema allegorico dantesco che viene utilizzato come motore intellettuale del presente perché ha ancora molto da dire al mondo contemporaneo.

Da una cinquantina d'anni insomma, in terra di saudade, ci si avvale di Dante e della sua opera oltre che come simbolo d'amore idealizzato e della conseguente saudade, anche come simbolo di protesta e di rivoluzione (come nell'album dei Mutantes e dei Sepultura). E come in lingua portoghese l'espressione divina comedia è antonomasia di disordine e confusione, concetti legati nell'inconscio collettivo all'Inferno e alla sua rappresentazione, così attraverso le scelte del genere musicale, dallo psichedelico al metal, si fa passare il concetto di totale estraneità alla società, verso la quale è possibile solo esprimere il proprio dissenso. Ma non un dissenso fine a se stesso, dal momento che la musica e il movimento tropicalista hanno inciso fortemente sulla storia del Brasile e l'identificazione del dissenso con un autore come Dante non fa altro che ritrovare la saudade come esperienza e non solo speranza di un mondo migliore.

E forse non sorprende che l'opera dantesca sia stata l'oggetto di una serie di album di un band italiana di rock progressivo, i Metamorfosi, che nel 1973 hanno pubblicato "Inferno" (Vedette), il primo di una serie di tre dischi dedicati alla Divina Commedia in cui i peccatori e gli ambienti infernali sono riferimenti agli aspetti critici della società, richiamando, già nei titoli dei brani, spaccio di droga, politica, razzismo. ${ }^{36}$

Per i musicisti brasiliani degli anni '7o la saudade è un percorso che va dal dissenso nella musica all'esperienza concreta del movimento di protesta, ritor-

36. Il secondo album, "Paradiso", è uscito dopo una trentina d'anni, nel 2004, mentre il terzo disco, "Purgatorio", viene annunciato sul sito ( http://www.metamorfosi.me / main.htm) come in fase di preparazione. Anche altri artisti italiani pop e cantautori hanno dedicato dei brani o interi album a personaggi della Commedia dantesca: Caronte (The Trip), Pia come la vedo io (Gianna Nannini), Filippo Argenti (Caparezza), ma come nel caso dei Metamorfosi, si tratta di casi molto diversi rispetto al movimento culturale e sociale del Tropicália e dell'“invenzione" brasiliana della musica pop. 
nando per altra via alla società e cioè attraverso il "prodossumo" che coinvolge nel movimento artistico e nel mercato musicale la società stessa che aveva causato il dissenso. Un labirinto, come Lourenço l'ha definita (v. supra) ma, parafrasando Tabucchi, una saudade del presente che, come saudade attiva e come il desio dantesco, è la tensione a continuare il viaggio verso il futuro, lo sprone alla trasformazione e al mutamento di rotta, l'energia della spinta che si ritrova in quegli anni anche se con modalità diverse nel panorama musicale italiano.

Siamo alla fine della navigazione. Il viaggio poetico della navicella dantesca sul mare del desio e della saudade non pretende di essere esaustivo, giacché il fatto stesso di navigare lascia ogni operazione in fieri. Per Pessoa "navegar é preciso, viver não é preciso" e per "nós, argonautas" 37 ogni navigazione musicale, artistica, intellettuale, sociale - verso "miglior'acque" è, in fondo, saudade, ed è saudade de viver.

\section{BibliografiA}

Andrade, O. de, 1928, Manifesto Antropófago, «Revista de Antropofagia», anno I, n. I, http://www.tanto.com.br/manifestoantropofago.htm (rev. 7/10/2016).

Bortolotti, M., 2013, A divina tragédia de Belchior, «Época», 28 dicembre 2013, Editorial Globo, Rio de Janeiro. http://www.epoca.globo.com/vida noticia/20I3/I2/divina-tragedia-de-bbelchiorb.html (rev. 7/Io/2016).

Calado, C., 1996, A Divina Comédia dos Mutantes, Editora 34, São Paulo, https:// books.google.it/books?id=Kx4mLGPSBMAC\&printsec=frontcover\&hl=it\#v=on epage\&q\&f=false (rev. 7/1o/2016).

Campos, A. de, 1978, Balanço da bossa e outras bossas, Perspectiva, São Paulo.

Costa, R. M. da, 2006, Tradição e vanguarda nos arranjos de Rogério Duprat para a Tropicália, Universidade Federal do Estado do Rio de Janeiro, Rio de Janeiro. Tesi finale di Master in Musica, http://www.rodrigomarconi.com/wp-content/uploads/20I6/oI/disserta\%C3\%A7ao-mestrado.pdf (rev. 7/Io/20I6).

D’Agostino, P., 2006, Introduzione, in Lourenço E., Mitologia della saudade, Orientexpress, Napoli, pp. 7-2I.

Fenzi, E., 20II, La canzone di Dante 'Amor che movi tua vertù dal cielo': una teoria anticavalcantiana sulla natura d'amore, in Grupo Tenzone, Amor che movi tua vertù dal cielo, Departamento de Filología Italiana UCM - Asociación Complutense de Dantología, Madrid, pp. 15-59.

Goffman, K. - Joy, D., 2007, Contracultura através dos tempos: do mito de Prometeu à cultura digital, Ediouro, Rio de Janeiro.

37. Non possiamo passare sotto silenzio l'ottimo lavoro di un gruppo di giovani musicisti di Bari, "Os Argonautas", il cui primo album, Navegar é preciso (Digressione Music, 2012) ha ispirato in parte il presente lavoro. Il disco miscela sapientemente la saudade lusitana con l'ambiente culturale e musicale non solo italiano ma anche regionale e pugliese, passando attraverso la musica mediterranea e arabo-andalusa, in un lavoro di matrice sperimentale ma fortemente folk-acustico. 
Lourenço, E., $1992^{5}$ (prima ed. 1972), O labirinto da saudade, Dom Quixote, Lisboa, https://www.academia.edu/477I490/O_LABIRINTO_DA_SAUDADE_ EDUARDO_LOUREN\%C3\%87\% (rev. 7/10/2016).

—, 1999, Portugal como destino seguido de Mitologia da saudade, Gradiva, Lisboa.

—, 2006, Mitologia della saudade, a cura di P. D’Agostino, Orientexpress, Napoli.

—, 2013, Il labirinto della saudade. Portogallo come destino, a cura di R. Vecchi e V. Russo, Diabasis, Parma.

Michaëlis de Vasconcelos, C., I990, A Saudade Portuguesa, Estante Editora, Aveiro.

Motta, N., 2009, Noites tropicais. Solos, improvisos e memórias musicais, Objetiva, Rio de Janeiro.

Nunziata, F., 2006, Recensione a Sepultura, Dante XXI, "Ondarock», Io dicembre 2006, s/l., http://www.ondarock.it/recensioni/2006_sepultura.htm (rev. 7/10/2016).

Folha, 1968, Música, pesquisa e audacia: o tropicalismo se define pelo debate, "Folha da Tarde», 7 giugno I968, Sao Paulo, http://tropicalia.com.br/eubioticamenteatraidos/polemica/o-tropicalismo-se-define-pelo-debate (rev. 7/1o/2016).

Paiva, J. E. R. de, 2006, "Os Mutantes: Hibridismo Tecnológico Na Música Popular Brasileira Dos Anos 6o / 70", Universos da música. Cultura, sociabilidade e a politica de práticas Musicais, Anais do III Congresso da Associaçáo Brasileira de Etnomusicologia, s/l, pp. 497-502.. http://abetmusica.org.br/conteudo. php? \&sys=downloads (rev. 7/Io/2016).

Pessoa, F., I997, Quadras ao gosto popular, edição de Luís Prista, IN-CM, Lisboa.

—, 2005, http://www.fpessoa.com.ar/, sito a cura di Sebastián Santisi, Buenos Aires (rev. 7/10/2016).

—, 20I0, Livro do desassossego, Clube de autores, Joinville.

Plutarco, 1996, Vite parallele. Agesilao e Pompeo, Biblioteca Universale Rizzoli, Milano.

Russo, V., 2013, Trattato portatile della saudade con Pessoa sullo sfondo, in Aa. Vv., Sconfinamenti. Omaggio a Davide Bigalli, Bietti, Milano.

Tabucchi, A., 2003, Studi di Letterature Comparate in onore di Remo Ceserani-Letture e Riflessioni Critiche (vol. I), a cura di Mario Domenichelli [et al.], Vecchiarelli Editore, Roma, pp. 347-354, http://www.centrostudilusofoni.unibari.eu/ index. php? option=com_content $\&$ task=view $\&$ id $=42 \&$ Itemid $=$ I $\&$ lang=italian $($ rev. 7/10/2016).

UOL, 1968, Passeata de Cem Mil, http://wwwi.uol.com.br/rionosjornais/rj49.htm (rev. 7/10/2016).

Varela-Portas de Orduña, J., 20I4, Il corpo eterodosso di Dante Alighieri, in Cattermole, C., de Aldama, C., Giordano, C. (eds.), Ortodossia ed eterodossia in Dante Alighieri, Atti del Convegno di Madrid (5-7 novembre 20I2), Ediciones de La Discreta, Alpedrete, pp. 325-340.

Veloso, C., 1997, Verdade Tropical, Companhia das Letras, São Paulo.

Witzel, L. - Costa, J. C., 20I3, "Os Mutantes: O mito que permeia a história ou a contracultura que salva, amém-te!», Anais do I Congresso Internacional de Estudos do Rock, Unioeste, Cascavel. http://www.congressodorock.com.br/evento/ anais/2013/artigos/5/artigo_simposio_6_Ludmila_Witzel.pdf (rev. 7/1o/2016).

Wiznitzer, A., s/d, Recensione a Divina Comédia ou Ando Meio Desligado, "All Music», San Francisco, http://www.allmusic.com/album/a-divina-com\%C3\%A9dia-ouando-meio-desligado-mwoooo231423 (rev. 7/Io/20I6). 


\section{DisCOGRAFIA}

Veloso, C., 1969, Os Argonautas, in Id., Caetano Veloso, Philips.

—, I978, Sampa, in Id., Muito, Universal Music Japan.

Os Mutantes, 1970, Divina Comédia ou Ando Meio Desligado, Polydor.

Belchior, 1991, Divina comédia humana, in Id., Divina comédia humana, Movie Play.

Scracho, 2007, Divina Comédia, in Id., A grande bola azul, Etichetta indipendente.

Sepultura, 2006, Dante XXI, Steamhammer / SPV.

Metamorfosi, 1973, Inferno, Vedette.

-, 2004, Paradiso, Progressivamente.

\section{Sitografia}

Belchior: https://www.vagalume.com.br/belchior (rev. 7/10/20I6).

Metamorfosi: http://www.metamorfosi.me/main.htm (rev. 7/1o/20I6).

Os Argonautas: http://osargonautas.wixsite.com/osargonautas (rev. 7/ro/2016).

Os Mutantes: https://www.vagalume.com.br/os-mutantes/ (rev. 7/1o/20I6).

Terra em Transe: https://www.youtube.com/watch?v=bX4BLrGwWGQ (rev. I2/IO/2016).

Tropicália: http://www.tropicalia.com.br, http://www.caetanoendetalle.blogspot. it/2013/Io/1968-debate-tropicalista.html (rev. 7/Io/2016).

Scratcho: https://www.facebook.com/scrachooficial/, https://www.vagalume.com.br/ scracho/ (rev. 7/Io/2016).

Sepultura: http://www.sepultura.com.br/, https://www.vagalume.com.br/sepultura/ (rev. 7/10/2016).

Veloso: http://www.caetanoveloso.com.br/, https://www.vagalume.com.br/caetanoveloso (rev. 7/1o/2016). 